\title{
ANÁlise da Volatilidade Do ÍNDICE BOVESPA: UM ESTUDO EMPÍRICO UTILIZANDO MODELOS DA CLASSE ARCH
}

\author{
Luiz Eduardo Gaio \\ Graduando em Administração pela Universidade Federal \\ de Lavras, Bolsista PET/SESU e Pesquisador pelo \\ Grupo de Estudos em Finanças Empresariais. \\ lugaio@yahoo.com.br
}

Gabriel Rodrigo Gomes Pessanha

Graduando em Administração pela Universidade Federal de Lavras e Bolsista CNPq. gabrielrgp@yahoo.com.br

Denis Renato de Oliveira

Mestrando em Administração de Organizações pela FEA-RP/USP

denisufla@yahoo.com.br

Leiziane Neves de Ázara

Bacharel em Administração pela Universidade Federal de Lavras leizianeazara@yahoo.com.br

\section{Resumo}

A utilização de estudos sobre volatilidade como instrumento de orientação de investimentos e classificação de riscos tem sido uma estratégia muito utilizada no mercado de capitais. Este artigo faz uma análise empírica da volatilidade dos retornos do índice Bovespa por meio de modelos da classe ARCH. Os resultados empíricos sugerem fortes sinais de persistência e assimetria da volatilidade dos retornos da série. Além disso, todos os modelos da classe ARCH estimados tiveram um bom desempenho, destacando o modelo EGARCH $(1,1)$, gaussiano, apresentando o melhor ajuste considerando os critérios de qualidade. Os resultados sugerem, também, a diversificação da carteira de investimentos como um importante instrumento da administração do risco e de suas operações comerciais, uma vez que, os choques negativos e positivos têm impactos diferenciados sobre a volatilidade dos retornos.

Palavras-chave: Volatilidade, índice Bovespa, modelos ARCH, Mercado de Capitais, Econometria.

\begin{abstract}
The use of studies about volatility as an instrument to guide investments and risk classification has been an usual strategy in stock market. This paper does an empirical analysis of the volatility in Bovespa index returns with ARCH models. The empirical results suggest that there are strong persistence and skewness signals volatility in series returns. Besides, all $A R C H$ models estimated had a good performance, outstanding the EGARCH(1,1), gaussian model, as there are which better adjusted according to adjust quality criterion. The results suggest, too, that the portfolio diversification is an important instrument to risk management of its commercial operations, because the negative and positive shocks have different impacts on the volatility
\end{abstract}

Key words: Volatility, Bovespa index, ARCH models, Stock Market, Econometrics. 


\section{INTRODUÇÃO}

Durante duas décadas a volatilidade tem sido foco de pesquisas em séries temporais econométricas. Os estudos de volatilidade têm se limitado também em questões de estimação, inferência estatística e especificação de modelos. A pesquisa de volatilidade tem contribuído grandemente para a compreensão de questões em finanças econômicas, especialmente no que se refere à mensuração de incertezas, tal como alocações de portifólio, precificação de opções e administração de risco.

A volatilidade pode ser destacada como uma das ferramentas mais importantes para quem atua no mercado de opções, devido, principalmente, à importância dada à observação de sua direção e velocidade de movimentação. Em certo sentido, a volatilidade é uma medida da velocidade do mercado e, teoricamente, o número "volatilidade" associada ao preço de uma mercadoria é a variação de preço referente a um desvio padrão, expresso em porcentagem, ao fim de um período de tempo. Portanto, se uma ação tem hoje um preço de 50 unidades monetárias, com volatilidade de $20 \%$ ao ano, espera-se que esta ação daqui a um ano, em média, esteja situada entre 40 e 60 unidades monetárias.

A correta análise da volatilidade se torna importante não só para o esboço estratégico na administração de ativos como também para a captação dos momentos de incerteza no mercado, ou seja, quanto maior o grau de incerteza frente às crises ou fatores exógenos maiores serão as variações nos preços e na variância dos retornos, implicando desta forma, em grandes possibilidades de ganhos e/ou perdas. Assim sendo, o correto gerenciamento de risco de uma carteira de investimentos passa pela boa previsão das oscilações de preços dos ativos no mercado (MORAIS; PORTUGAL, 1999).

Ao remeter o estudo da volatilidade para o índice Bovespa (IBOVESPA), cuja finalidade se define em indicar comportamento médio das ações de mercado, procura-se na sua composição aproximar ao máximo da real configuração das negociações à vista (lote-padrão), verificando a variação dos indicadores de desempenho médio das cotações do mercado de ações brasileiro e permitindo, desta forma, uma visualização clara das mudanças ocorridas nos preços dos principais papéis negociados na bolsa, além do real subsídio dado ao investidor, no que se refere às informações necessárias para o gerenciamento e posicionamento de mercado.

Assim sendo, o presente artigo trata apenas dos modelos de especificação da volatilidade determinística, apresentando um estudo empírico do processo de volatilidade do retorno do IBOVESPA e analisando duas características: a persistência de choques e a assimetria na volatilidade. Para tanto serão utilizadas como ferramentas estatísticas de estimação e análise da volatilidade os modelos da família ARCH (GARCH; EGARCH;
TARCH), objetivando contribuir não apenas para o preenchimento de parte da lacuna observada nos estudos sobre volatilidade de ações na Bolsa de Valores em São Paulo, como também, apresentar um estudo científico de análise da volatilidade do IBOVESPA, a fim de que o mesmo seja aplicado a processos de gerenciamento de riscos e controle de garantias no referido mercado.

\section{PROCEDIMENTOS METODOLÓGICOS}

Utilizou-se neste trabalho as cotações diárias do fechamento do IBOVESPA na Bolsa de Valores de São Paulo (BOVESPA), observadas durante o período de 03 de janeiro de 2000 a 29 de dezembro de 2005, perfazendo um total de 1491 observações. Os dados foram coletados junto ao site da Bovespa e a escolha da datas inicial e final para a amostra foram arbitradas pelos pesquisadores, não possuindo, portanto, nenhum significado especial.

Com base na premissa de log-normalidade dos preços, a série do índice Bovespa foi transformada por intermédio da equação $r_{t}=\ln \mathrm{P}_{t}-\ln \mathrm{P}_{\mathrm{t}-1}$, resultando em uma série de retornos do IBOVESPA (1490 observações). Dessa forma, as séries de retornos substituiu as séries originais do IBOVESPA tornando-se dados de entrada para os testes estatísticos e para os modelos de predição de volatilidade. Conforme Tsay (2002) existem duas razões principais para se trabalhar com retornos, e não preços: a primeira é que para investidores médios, o retorno de um ativo é um sumário completo e independente da escala da oportunidade de investimento e, a segunda, que séries de retornos são mais fáceis de manipular que séries de preços, dado que as primeiras têm propriedades estatísticas mais tratáveis.

A primeira etapa da análise empírica se constitui em verificar visualmente o comportamento da série temporal do índice Bovespa e sua estatística descritiva, a fim de verificar fatores como assimetria, caudas pesadas, excesso de curtose e normalidade na distribuição da série.

$\mathrm{Na}$ segunda etapa observa-se a verificação de estacionaridade da série de retorno do IBOVESPA através dos testes de raiz unitária, Dickey e Fuller Aumentado (ADF) e Phillips e Perron (PP).

A terceira etapa busca verificar a presença de heteroscedasticidade condicional auto-regressiva na série de retorno do IBOVESPA. Para esta verificação utiliza-se o teste ARCH-LM (ARCH - Lagrange Multipliers). A presença de heteroscedasticidade é algo fundamental quando se utiliza modelos da classe ARCH.

Depois de testada a presença de heteroscedasticidade condicional, parte-se para a quarta etapa, na qual se efetua a modelagem dos dados. Ressalta-se ainda, segundo Goulart et al. (2005), que anterior à fase de descrição dos modelos da família ARCH é importante destacar em sua utilização, a exigência da definição em conjunto da equação para a média condicional, uma vez que, os erros apu- 
rados na equação da média são os insumos necessários à operacionalização dos cálculos da volatilidade condicionada. Alexander (1998) postula que, quanto mais parcimoniosa for a definição da equação da média condicional, melhor será possível avaliar o desempenho do modelo. Isto posto, utilizou-se, primeiramente, os modelos ARMA (autoregressive movie average) para modelar as dependências temporais presentes na série. Posteriormente, os modelos ARCH foram ajustados aos erros quadráticos do modelo ARMA de modo que se extraísse a volatilidade da série.

Por fim, a quinta etapa constitui-se da análise e seleção do modelo que melhor se ajustou à série de volatilidade do índice Bovespa. Como medida de avaliação e seleção do melhor modelo para série utilizou-se o Critério de Informação de Akaike, o valor da máxima log-verossimilhança, o erro padrão do modelo e os resultados do teste ARCH-LM para os lags 1, 5 e 10, que busca verificar a eliminação completa da heteroscedasticidade condicional. O critério Akaike é especificado conforme a função:

$$
\text { Akaike }=-2\left(\frac{\log \left(L_{T}(\Theta)\right)}{n}\right)+2\left(\frac{k}{n}\right)
$$

Onde: $\log \left(L_{T}(\Theta)\right)$ denota o valor da log verossimilhança; n é o número de observações; $\mathrm{k}$ o número de parâmetros.

Para o ajustamento e análise da volatilidade dos retornos do índice Bovespa utilizou-se o software estatístico $\mathrm{Ox} \circledast$ com o pacote específico G@RCH 2.3, sugeridos por Laurent e Peters (2002). Sugere-se, para maiores detalhes sobre o software, consultar Doornik (2001).

\subsection{Modelos de Séries Temporais}

Uma série temporal é definida por Box et al. (1994) como um conjunto de observações geradas seqüencialmente no tempo. Essencialmente, existem dois enfoques usados na análise de séries temporais e ambos possuem o objetivo de construir modelos para as séries, com propósitos determinados. No primeiro, a análise é realizada no domínio da freqüência e os modelos propostos são não-paramétricos, como, por exemplo, a análise espectral. No segundo, a análise é conduzida no domínio temporal e os modelos propostos são paramétricos, para os quais o número de parâmetros envolvidos na análise é finito, como, por exemplo, os modelos ARIMA (MORETTIN; TOLOI, 2004). Nos dois enfoques, os modelos devem ser simples e parcimoniosos, ou seja, deve-se envolver o menor número de parâmetros possível.

A análise de séries temporais pode ser focada, conforme Morettin e Toloi (2004), nos seguintes propósitos:

- na investigação do mecanismo gerador da série temporal;

- na previsão de valores futuros da série;

- apenas na descrição do comportamento da série e;

- na procura de periodicidade relevantes nos dados.

Segundo Alexander (2005), o objetivo da análise de séries temporais é encontrar o modelo estatístico que melhor possa se adequar aos dados, bem como utilizar esse modelo para a previsão. Desse modo, pode-se permitir que as variáveis falem por si mesmas, ou seja, sem as restrições da teoria econômica e da teoria das finanças.

Portanto, a construção de um modelo que descreva os movimentos passados de uma variável de determinada série temporal, proveniente de análises sistemáticas do comportamento dessa série, é capaz de predizer movimentos futuros dessa variável.

\subsubsection{Modelos ARIMA}

Os modelos ARIMA (Autorregressivo - Integrados - Médias móveis) foi formulado por Box e Jenkins (1976) e baseiam-se na idéia de que séries temporais nãoestacionárias podem ser modeladas a partir de d diferenciações e, também, da inclusão de componentes autoregressivos e médias móveis. (BRESSAN; LIMA, 2002).

Sendo \{Y\} um processo que pode ser descrito através de uma modelagem ARIMA(p,d,q) da seguinte forma:

$\phi_{1}(B) z_{i}=\theta+\theta(B) a_{i}$

onde: $z=\left\{\begin{array}{c}Y \\ (1-B)\end{array}\right.$

$Y$ para um processo estacionário $(\mathrm{d}=0)$ e $(1-B)^{d}$ se o processo não é estacionário $(\mathrm{d}>1)$.

Ao se considerar a diferenciação de $Y$ tem-se um modelo ARIMA(p,d,q) com:

$\phi(B)(1-B) Y=\theta+\theta(B) a$

onde: $\phi_{0}(B)=1-\phi_{1} B-\ldots-\phi B$ é o operador autorregressivo $A R(p)$, e $\theta(B)=1-\theta_{1} B-\ldots-\theta B^{g}$ é o operador de média móvel MA(q) e $a$ é processo ruído branco.

Vale lembrar que $Y$ será o retorno do Ibovespa, ou seja, $Y=r$, caso a série de retorno não seja um ruído branco, necessitando então de uma modelagem temporal utilizando o modelo ARIMA(p,d,q). Porém, nos casos em que a série de retorno se apresenta como um ruído branco, ocorrendo geralmente em mercados eficientes, o ajustamento de modelos autorregressivos e médias móveis não ocorrem, partindo-se direto para os modelos de 
volatilidade.

\subsubsection{Modelos de Volatilidade}

Os primeiros estudos de modelagem de volatilidade foram desenvolvidos por Engle (1982) e, segundo o mesmo autor, um processo denominado ARCH (autoregressive conditional heteroskedasticity) expressa a variância condicional como sendo uma defasagem distribuída do quadrado dos retornos passados, ou seja, a idéia básica foi que o retorno na série é não-correlacionado serialmente, mas a volatilidade (variância condicional) depende de retornos passados por meio de uma função quadrática. Podemos definir um modelo $\mathrm{ARCH}(\mathrm{p})$ por:

$$
y=\sqrt{\sigma^{2}} \varepsilon
$$

$\sigma^{2}=\alpha_{0}+\sum_{-1}^{p} \alpha y_{-}^{2}$

onde: $y$ é o retorno; $\boldsymbol{\varepsilon}$ é uma seqüência de variáveis aleatórias independentes e identicamente distribuídas (i.i.d.) com média zero e variância um.

Morettin e Toloi (2004) afirmam que, na maioria das vezes, os erros dos modelos de volatilidade condicional assume uma distribuição normal ou uma distribuição t de Student, sendo $\varepsilon_{t} \sim \mathrm{N}(0,1)$ ou $\varepsilon_{t} \sim t_{v}$ (t de Student com $v$ graus de liberdade) respectivamente.

Conforme afirmado anteriormente, caso a série de retorno apresentem uma autocorrelação serial, os modelos de volatilidade não se aplicam nas séries de retornos propriamente ditas, e sim, nos resíduos das séries após o ajustamento de um modelo ARIMA. Em outras palavras, os modelos de volatilidade não serão aplicados em $\gamma_{1}$ (série de retorno) e sim na série $a_{\text {(resíduo dos mode- }}$ los ARMA).

Conforme Mol (2003) este modelo de variância condicional possui algumas propriedades desejáveis. Em primeiro lugar, por meio da técnica de decomposição de erros de predição é possível construir a função de verossimilhança, fazendo-se possível a estimação dos parâmetros pelo método de máxima verossimilhança. Esta propriedade é importante porque estes estimadores possuem distribuições conhecidas que viabilizam a execução de testes de hipóteses diversos e, além disso, é possível provar que este modelo implica em uma distribuição não condicional com "caudas pesadas" para os retornos.

A proposição original elaborada por Engle (1982) mereceu, ao longo dos anos, extensos debates e diversos aperfeiçoamentos. O primeiro e mais significativo foi introduzido por Bollerslev (1986) ao propor que a volatilidade condicionada era uma função não apenas dos quadrados dos retornos passados $\left(y_{i}^{2}\right)$, c omo também dos seus próprios valores $\left(\sigma_{-}^{2}\right)$, passando os modelos assim construídos a ser denominados Generalized ARCH
(GARCH). Em termos matemáticos, um modelo GATCH $(p, q)$ pode ser expresso como:

$\sigma^{2}=a+\sum_{-}^{n} \alpha y_{--}^{2}+\sum_{-}^{4} \beta \sigma_{--}^{2}+v_{i}$

onde: $\omega$ é a constante $v$, é um ruído branco $[\mathrm{N} \sim(0,1)]$.

A fim de se garantir que a variância condicional não seja negativa, e também a estacionariedade do processo, temos que $\omega>0 ; \alpha \geq 0$, para $i=1, \ldots, p ; \beta \geq 0$, para $\mathrm{j}=1, \ldots, \mathrm{q} ; \mathrm{e} \sum_{=}^{\beta} \alpha+\sum_{\alpha} \beta<1$

É interessante ressaltar, que apesar do modelo GARCH (p,q) captar corretamente diversas características observadas nas séries históricas de finanças, tais como a leptocurtose e o agrupamento de volatilidade, este não capta o efeito de alavancagem, uma vez que, a variância condicional é função apenas das magnitude das inovações e não dos seus sinais (BROOKS, 2002). A partir daí, surgiu a idéia de se criar modelos que tivessem a capacidade de captar a assimetria das respostas, tais como os modelos EGARCH, TARCH e APARCH.

O modelo EGACH (Exponential GARCH), proposto por Nelson (1991), foi uma inovação aos modelos de volatilidade uma vez que os choques na variância passam a ter efeito exponencial e não quadrático. Este mesmo modelo apresenta duas vantagens em relação ao modelo GARCH: a incorporação dos efeitos assimétricos de mercado aos modelos auto-regressivos de volatilidade condicional e a não imposição artificial de restrições aos parâmetros da equação, dada a sua formulação em termos logarítmicos.

$\mathrm{Na}$ forma simplificada o modelo EGARCH (p,q) pode ser expresso:

$\ln \left(\sigma^{2}\right)=a_{a}+\sum\left(a\left(\left|\frac{y}{\sigma}\right|-E\left|\frac{y}{\sigma}\right|\right)+y \frac{y}{\sigma}\right)+\sum\left(b \ln \left(\sigma^{2}\right)\right.$

onde: o parâmetro $\gamma$ permite um efeito assimétrico, e se $\gamma_{1}=0$, é um indicativo de ausência de assimetria na volatilidade. Neste sentido, um procedimento de teste para o efeito da assimetria na série é checar a significância de $\gamma_{1}$ no modelo. Se $\gamma_{1}$ é estatisticamente diferente de zero, evidencia-se um impacto diferenciado de choques negativos e positivos na volatilidade. Se $\gamma_{1}<0$, há presença do "efeito alavancagem". Neste modelo, a persistência de choques na volatilidade é medida pelo parâmetro b.

Segundo Bollerslev e Wooldridge (1992), estimadores de máxima verossimilhança dos parâmetros de um modelo GARCH podem ser utilizados assumindo-se inovações gaussianas, ainda que a verdadeira distribuição não seja gaussiana. Para que se possa conseguir a consistência necessária neste processo, utiliza-se a matriz de variância-covariância corrigida, proposta pelos autores. Neste trabalho, adotou-se a correção proposta por 
Bollerlev e Wooldridge (1992), uma vez que as distribuições de séries de retorno apresentam-se de forma leptocúrticas.

Um modelo mais simples para a captação do comportamento assimétrico da volatilidade nas séries financeiras foi apresentado por Glosten, Jagannathan e Runkle (1993) e, posteriormente, implantado por Zakoian (1994), denominado TARCH (Threshold ARCH). Esse novo modelo é um caso particular do modelo ARCH não-linear, e a volatilidade agora, segue a forma funcional:

$\sigma^{2}=a+\sum_{-}^{n} \alpha y_{-}^{2}+p_{-2}^{2} d_{--}+\sum_{-}^{4} \beta \sigma_{--}^{2}+\nu$

onde: $d_{t-1}$ é uma variável dummy que assume o valor igual a 1 se $y_{t-1}<0$ (más notícias, e valor igual a 0 se $y_{t-1}>0$ (boas notícias).

Destacando que, como no modelo GARCH, as condições para a não negatividade e estacionariedade são $\omega>0 ; \alpha \geq 0$, para $\mathrm{i}=1, \ldots, \mathrm{p} ; \beta \geq 0$, para $j=1, \ldots$, q; e $\sum \alpha+\gamma \geq 0$ (BROOKS, 2002).

\section{ANÁLISES E DISCUSSÃO}

Apresenta-se nesta seção a discussão dos resultados da análise da volatilidade do retorno do índice Bovespa, utilizando modelos da família ARCH (Autoregressive Conditional Heteroskedasticity) como ferramenta estatística de estimação de volatilidade.

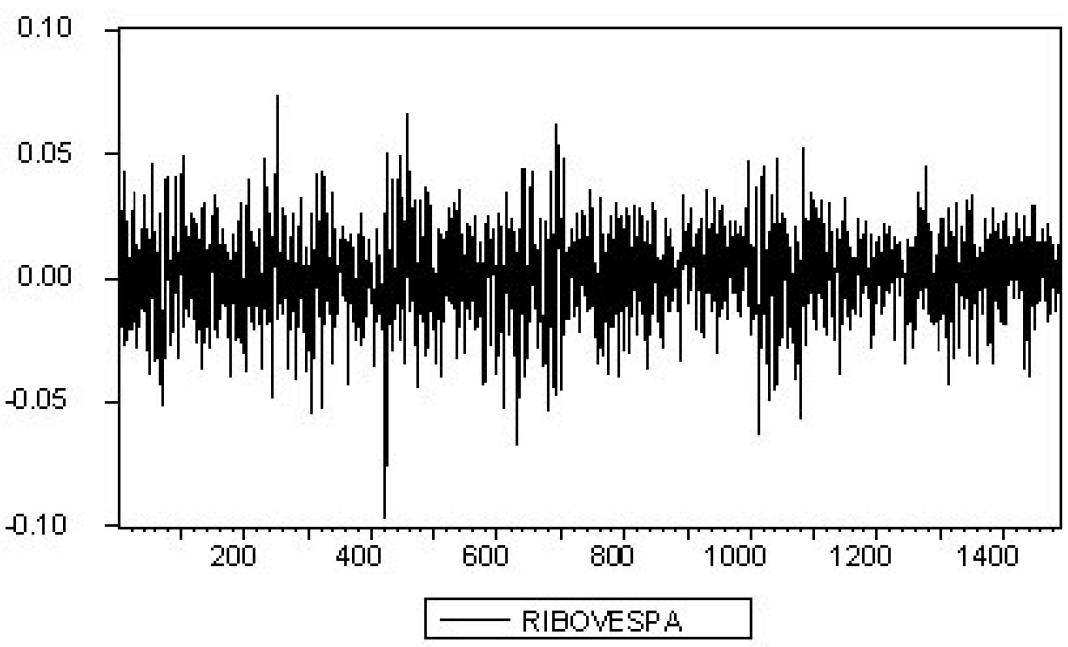

Figura 2: Série de retornos diários IBOVESPA - 03 de janeiro de 2000 a 29 de dezembro de 2005

Fonte: Dados da pesquisa

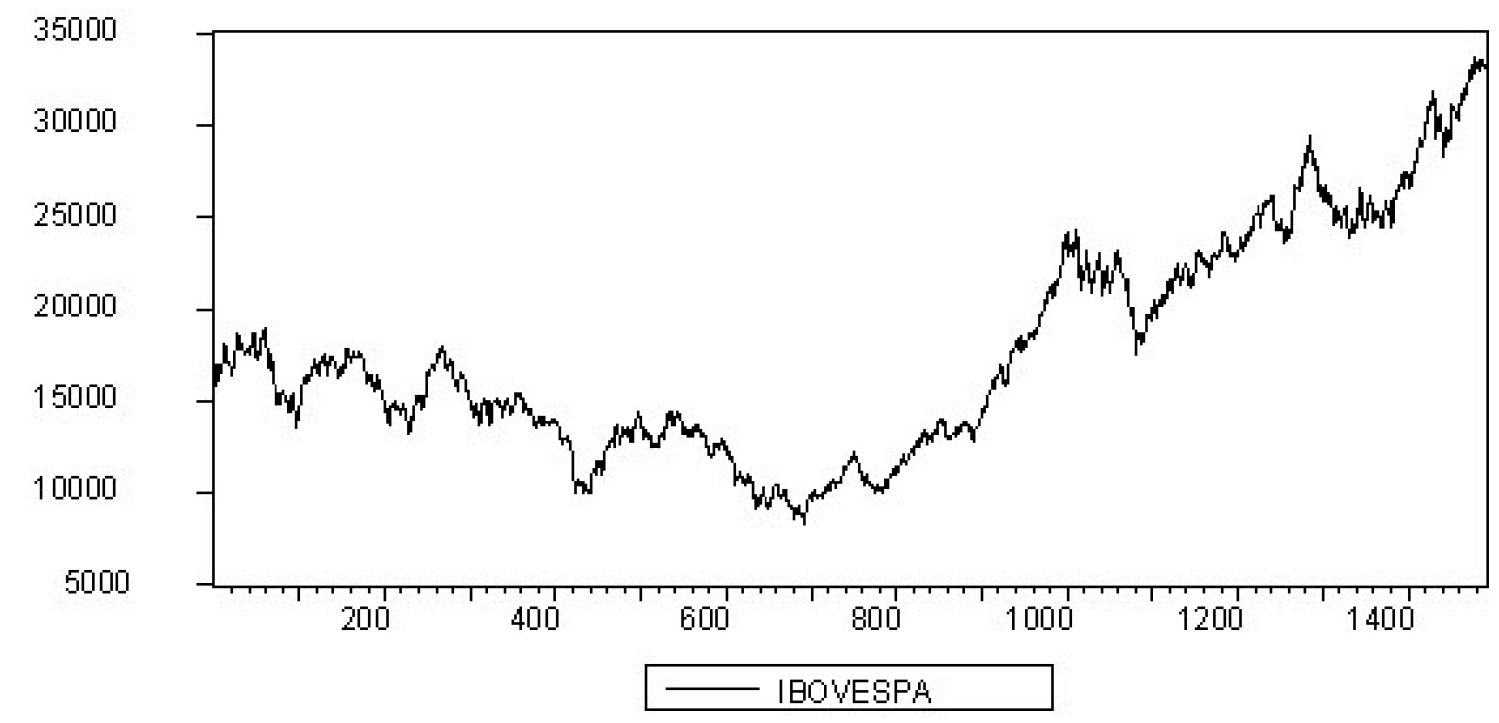

Figura 1: Série de cotações diárias IBOVESPA - 03 de janeiro de 2000 a 29 de dezembro de 2005.

Fonte: Dados da pesquisa 
ente de assimetria e excesso de curtose quantifica os desvios da distribuição normal e se define no trabalho de Zhou (2000).

Tabela 1. Estatísticas descritivas

\begin{tabular}{ll}
\hline Estatisticas & IBONESPA \\
\hline Média & 0,000457 \\
Me diana & 0,000826 \\
Márimo & 0,073356 \\
Minimo & $-0,096286$ \\
Desvio padrão inconcticional & 0,018813 \\
Assimettia & $-0,227214$ \\
Guttose & 3,870199 \\
Jatque-Beta & 59,83291 \\
Probability & 0,000000 \\
\hline
\end{tabular}

Fonte: Dados da pesquisa

De acordo com a Tabela 2 podemos identificar que a série de retornos do IBOVESPA é estacionária e não contêm raízes unitárias. Os testes foram realizados através dos critérios Dickey-Fuller Aumentado (ADF) e PhillipsPerron (PP), propostos respectivamente por Dickey e Fuller (1979) e Phillips e Perron (1988).

Tabela 2: Teste de estacionaridade para a série de retornos do IBOVESPA.

Tes te para raiz unitaria Retorno de IBOVESPA

\begin{tabular}{ll}
\hline Dickey \& Fullex (ADF) & $-37,26738$ \\
Phillipe \& Perron (P-) & $-37,26998$ \\
\hline
\end{tabular}

Fonte: Dados da pesquisa

Notas: Os valores críticos de significância de 1\%, 5\% e 10\% são $3,44,-2,86$ e $-2,57$, respectivamente ADF e PP testes são calculados pela estatística $t$

A tabela 3 contém algumas estimativas dos coeficientes das funções de autocorrelação e de autocorrelação parcial para os retornos e retornos quadráticos do IBOVESPA.
Tabela 3. Estimativa dos coeficientes de autocorre-lação e autocorrelação parcial para os retornos e retornos quadráticos.

\begin{tabular}{|c|c|c|c|}
\hline \multicolumn{2}{|c|}{ Fetotmos } & \multicolumn{2}{|c|}{ Retoxmos Quadratio os } \\
\hline al $\left(\mathrm{p}^{1}\right)^{+}$ & $0,038(0.038)$ & al $\left(\mathrm{p}^{1}\right)^{+}$ & $0,049(0,049)$ \\
\hline$a 2(\mathrm{p} 2)$ & $-0,026(-0,027)$ & $\mathrm{d}(\mathrm{p})$ & $0,15,4(0,152)$ \\
\hline as (po) & $-0,019(-0,017)$ & a) (pos) & $0,108(0,097)$ \\
\hline $\mathrm{A}_{4}\left(\mathrm{p}_{4}\right)$ & $-0,010(-0,009)$ & $\mathrm{a}_{4}\left(\mathrm{P}_{4}\right)$ & $0,116(0,089)$ \\
\hline as (ps) & $-0,017(-0,017)$ & as (ps) & $0,022(-0,015)$ \\
\hline $3 / \sqrt{T}$ & $0,0,52$ & $2 / \sqrt{T}^{:+=k}$ & 0,052 \\
\hline
\end{tabular}

Fonte: Dados da pesquisa

$* a_{i}$ e $p_{i}$ representam os coeficientes de autocorrelação e autocorrelação parcial.

** Limite assintótico da função de autocorrelação

Os coeficientes de autocorrelação estimados para as séries de retornos e retornos quadráticos revelaram um padrão previsível para a média condicional dos retornos do IBOVESPA, evidenciando também a presença de efeitos ARCH na volatilidade do mesmo. Objetivando confirmar tal evidência, efetuou-se o teste do tipo Multiplicador de Lagrange - ARCH LM test, proposto por Engle (1982) nos resíduos dos modelos AR ajustados para a média condicional dos retornos. Os p-valores do teste são reportados na Tabela 4 .

Tabela 4. Teste de heteroscedasticidade para a série de retornos do IBOVESPA - ARCH-LM

\begin{tabular}{cc}
\hline \multicolumn{2}{c}{ Fiesiduos / AR (1) } \\
\hline Lag & p-valox \\
\hline 1 & 0,0000 \\
5 & 0,0000 \\
10 & 0,0000 \\
\hline
\end{tabular}

Fonte: Dados da pesquisa

Em relação à heteroscedasticidade da série, conforme visto no teste ARCH-LM, os p-valores observados nas estatísticas indicam uma forte presença de autocorrelação dos resíduos quadráticos da série de retornos. Dessa forma, os testes sugerem a rejeição da hipótese de homoscedasticidade na série de retornos do IBOVESPA. 
Assim sendo, ajustou-se vários modelos para a média condicional da série de retorno, estabelecendo como melhor modelo um AR (1) para todos os modelos da classe ARCH na variância especificada. Os parâmetros dos modelos foram estimados pelo procedimento de Newey e West (1987), o que os tornou robustos à heterocedasticidade e autocorrelação no processo do resíduo.

O passo seguinte consistiu na modelagem da volatilidade dos retornos condicionais do IBOVESPA levando em consideração o padrão heterocedástico da volatilidade. Este fato foi implementado ajustando-se os modelos GARCH, EGARCH e TARCH à volatilidade dos modelos já ajustados para a média condicional dos retornos. Ao adotar a hipótese de normalidade dos resíduos dos modelos ajustados para a média condicional do retorno na estimação dos modelos, considerando ainda o fato estilizado "caudas pesadas", adotou-se a matriz de variância-covariância corrigida proposta por Bollerslev e Wooldridge (1992) no procedimento de estimação dos modelos da família ARCH.

Os resultados da estimação para a média condicional e a volatilidade dos retornos IBOVESPA estão reportados na Tabela 5. Pode-se observar a princípio, que os choques na série de retorno do IBOVESPA terão efeitos por vários períodos na sua volatilidade, ou seja, há uma persistência de choques na volatilidade, uma vez que a soma dos parâmetros $\alpha$ e $\beta$ no modelo $\operatorname{GARCH}(1,1)$ e o valor do termo $\ln \left(\sigma^{2}-1\right)$ foram próximos, sendo de 0,97 e 0,96 respectivamente. Outro ponto interessante a ser observado é a assimetria na volatilidade dos retornos, cuja presença pode ser evidenciada no modelo EGARCH(1,1), uma vez que o coeficiente do termo $\varepsilon_{-1} / \sigma_{-1}$ mostrou-se significativamente diferente de zero aos níveis de significância, ou seja, choques positivos e negativos têm impacto distinto sobre a volatilidade, porém, não há evidência de efeito alavancagem. O modelo TARCH $(1,1)$, também, confirma a assimetria evidenciada pelo modelo EGARCH(1,1), posto que o parâmetro associado ao termo $d_{-} \varepsilon^{2}-$ mostrou-se significativamente diferente de zero e também não fornece evidência do efeito alavancagem.

Ao analisar os indicadores da qualidade do ajuste, conforme apresenta a tabela 6, pôde-se perceber que todos os modelos ajustados para a série de retornos do IBOVESPA tiveram um desempenho bem favorável, porém o modelo que se destacou foi o modelo EGARCH. Uma possível hipótese para o fraco desempenho do modelo GARCH é a assimetria dos retornos, a qual não é capturada pelo modelo. Corrobora essa hipótese a supremacia dos modelos assimétricos (EGARCH; TARCH), segundo todos os critérios.
Tabela 5: Resultados da estimação dos modelos para os retornos do IBOVESPA.

\begin{tabular}{|c|c|c|c|}
\hline \multicolumn{4}{|c|}{ Estirnatiwas } \\
\hline \multicolumn{4}{|c|}{ Especific desca } \\
\hline Média & $\mathbf{A F}$ & $\mathbf{A R}$ & $\mathbf{A R}$ \\
\hline Cotistatite & - & - & - \\
\hline$A F(1)$ & $1,34(2,34)$ & $0,05(1,74)$ & $0,05(1,65)$ \\
\hline Wariância & GARCH & EGARCH & TARCH \\
\hline$a_{0}$ & $0,01(2,97)$ & $-0,38(-4,50)$ & $0,00(3,76)$ \\
\hline$E_{t-1}^{2}$ & $0,05(4,39)$ & & $-0,01(-0,97)$ \\
\hline$\sigma_{t-1}^{2}$ & $0,91(46,4)$ & & $0,92(53,4)$ \\
\hline$d_{t-1} e^{2}{ }_{t-1}$ & & & $0,10(5,21)$ \\
\hline$\left|z_{t-1}\right| / \mid \sigma_{t-1}$ & & $0,08(3,57)$ & \\
\hline$z_{t-1} / \sigma_{t-1}$ & & $-0,08(-6,37)$ & \\
\hline $\ln \left(\sigma^{2} t-1\right)$ & & $0,96(101,07)$ & \\
\hline
\end{tabular}

Fonte: Dados da pesquisa

Estatística $\mathrm{t}$ entre parênteses

Tabela 6: Medidas da qualidade do ajuste dos modelos estimados para a série de retornos do IBOVESPA.

\begin{tabular}{cccc}
\hline \multicolumn{4}{c}{ Qualidade do ajuste } \\
\hline It7dio adote s & GARCH & EGARCH & TARCH \\
lt1(L) & 3843,5 & $3861,8^{*}$ & 3859,5 \\
AIC & $-5,15$ & $-5,18^{*}$ & $-5,17$ \\
Ex+o Padro & 0,019 & $0,018^{*}$ & 0,019 \\
AFCH(1) & 0,269 & 0,155 & 0,191 \\
ARCH(5) & 0,178 & 0,172 & 0,176 \\
ARCH(10) & 0,271 & 0,308 & 0,265 \\
\hline
\end{tabular}

Fonte: Dados da pesquisa

Notas:

1. *denota o melhor modelo segundo cada critério.

2. $\ln (\mathrm{L})$ denota a máxima log-verossimilhança do modelo estimado.

3. AIC é o critério de informação de Schwartz.

4. Erro padrão denota o erro padrão do modelo.

5. ARCH(lag) denota que o teste efetuado até o lag entre parênteses.

De forma geral, pode-se observar que o mercado de ações na BOVESPA apresenta uma assimetria em sua volatilidade, uma vez que choques negativos tendem a se propagar de forma mais forte e em períodos mais extensos do que choques positivos, ou seja, a variação de preço no índice Bovespa é impactada pelos problemas fundamentais, como mudanças na política econômica. 
Portanto, as oscilações no índice Bovespa são ocasionados muito mais por resultados de problemas econômicos do que por características inerentes ao próprio mercado, uma vez que as modificações na volatilidade estão relacionadas, em última instância, com fatores exógenos. Dito de outra forma, a instabilidade nas políticas econômicas governamentais ou as modificações exógenas no volume de transações são muito mais importantes para justificar oscilações nesses mercados do que um possível caráter inerentemente instável do mercado.

A persistência e assimetria na volatilidade, sugerida pelos resultados obtidos, fornecem sugestões de que a utilização de estratégias no mercado acionário é um importante instrumento para a administração do risco de variação nos preços de suas operações comerciais, a fim de que os agentes deste mercado possam negociar de forma segura, diminuindo consigo a exposição à volatilidade noticiada e, portanto, a redução dos riscos envolvidos no mercado.

\section{CONCLUSÃO}

Realizou-se neste trabalho uma análise empírica da volatilidade dos retornos do índice Bovespa utilizando os modelos da classe ARCH como ferramenta de análise. Observou-se, desta forma, que o índice Bovespa é susceptível a reações de persistência e assimetria na sua volatilidade, ou seja, as variações dos retornos sofrem impactos diferenciados para boas e más notícias, o que pode ser comprovado pelos modelos GARCH, EGARCH e TARCH gaussianos. É interessante ressaltar que, mudanças na política governamental geram choques negativos e positivos que causarão grandes impactos nos preços futuros, repercutindo, então, por longos períodos.

Devem ser ressaltadas ainda, algumas limitações inerentes ao estudo como: o restrito número de observações decorrente do curto prazo de operação, a presença de pontos controversos e ainda inconclusos no meio acadêmico e o caráter probabilístico dos resultados. Dessa forma, os resultados obtidos não têm a pretensão de sinalizar uma análise minuciosa da volatilidade do índice Bovespa, mas sim, recolocar em discussão um tema de crucial importância para o gerenciamento de riscos e a precificação de operações no mercado de capitais brasileiro.

\section{REFERÊNCIAS}

ALEXANDER, C. Risk Management and Analysis. Measuring and Modeling Financial Risk. West Sussex: John Wiley. v. 1,1998 .

ALEXANDER, C. Modelos de Mercado: um guia para análise de informações financeiras. São Paulo: Bolsa de Mercadorias e Futuros, 2005.
BOLLERSLEV, T. “ Generalized autorregressive conditional heteroskedasticity". Journal of Econometrics, v.31, p.307-327, 1986

BOLLERSLEV, T; WOOLDRIDGE, J. M. “ Quasi-maximun likelihood estimation and inference in dynamic models with time-VaRying CoVaRiances". Econometric Reviews, v.11, n.2, p.143-172, 1992.

BOVESPA. Bolsa de Valores de São Paulo: IBOVESPA.

Disponível em: <http://www.bovespa.com.br/Pdf/Indices/ IBovespa.pdf $>$. Acessado em: 16 set. 2006.

BOX, G.; JENKINS, G. Time Series Analysis. Holden Day, San Francisco, Calif., 1976

BOX, G.; JENKINS, G.; REINSEL, G. C. Time Series Analysis, forecasting and control. 3. ed. Englewood Cliffs: Prentice-Hall, 1994, 598p.

BRESSAN, A. A.; LIMA, J. E. de. Modelos de previsão de preços aplicados aos contratos futuros de boi gordo na BMeF. Nova Economia, v. 12, n.1, p. 117-140, 2002.

BROOKS, C. Introductory Econometrics for Finance. Cambridge, UK: Cambridge University Press, 2002.

DOORNIK, J. A. Object-Oriented Matrix Programming using OX. 3rd end. London: Timberlake Consultants Press. 2001.

ENGLE, R. F. Autorregressive conditional heteroskedasticity with estimates of the variance of United Kingdom Inflation. Econometrica, v.50, n.4, p.987-1007, 1982.

GLOSTEN, L. R.; JAGANATHAN, R.; RUNKLE, D. E. On the relation between the expected value and volatility of the nominal excess returns on stocks. Journal of Finance, v. 48, p. 1779-1807, 1993.

GOULART, C. P.; AMARAL, H. F.; BERTUCCI, L. A.; BRESSAN, A. A. Previsão da volatilidade no mercado interbancário de câmbio. RAE - Revista de administração de empresas, Edição especial Minas Gerais, v. 45, p. 86-101, 2005.

GUIMARÃES, R. S. Volume de contratos futuros de soja negociados na Bolsa de Mercadorias e Futuros (BMeF). 2007, 99 p. Dissertação (Mestrado em Administração) - Universidade Federal de Lavras.

JARQUE, C. BERA, A. A Test for normality of observations and regression residuals. International Statistical Review, v.55, p.163-172, 1987.

LAURENT, S.; PETERS, J. O. GARCH 2,3: An Ox package for estimation and forecasting various ARCH models. Journal of Economic Surveys. Oxford, v. 16, p. 447-485, 2002.

MOL. A. L. R. Value at Risk como medida de risco da volatilidade dos ajustes diários em mercados futuros de café. 2003, 102 p. Dissertação (Mestrado em Administração) - Universidade Federal de Lavras.

MORAIS, I. A. C.; PORTUGAL, M. S. Modelagem e Previsão de volatilidade determinística e estocástica para a série do Ibovespa. Estudos Econômicos, São Paulo, SP, v. 29, n. 3, p.303-341,1999.

MOrettin, P. A.; TOLOI, C. M. C. Análise de séries temporais. São Paulo: Edgard Blücher, 2004.

NELSON, D. B. Conditional heteroskedasticity in asset returns: a new approach. Econometrica, v.59, n.2, p.347-370, 1991.

NELSON, D. Stacionarity and persistence in the $\operatorname{Garch}(1,1)$ Model. Econometric Theory, v.6, p.318-334,1991. 
NEWEY, W. ; WEST, K. A simple positive semi-definite, heroskedasticity and autocorrelation consistent covariance matrix. Econometrica, v.55, p.703-708, 1987.

PHILLIPS, P.C.B. ; PERRON P. Testing for a unit root in time series regression. Biometrika, v.75, p.335-346, 1988.

TSAY, R. S. The analysis of financial time series. New York: J. WILEY, 2002.

ZAKOIAN, A. Threshold heteroskedasticity models. Journal of Economic Dynamics and Control, v. 18, p. 931-955, 1994.

ZHOU, A. Modeling the volatility of the Heath-Jarrow-Morton Model: A Multi-Factor Garch Analysis. Illinois: University of Illinois at Urbana-Champaign, 2000. OFOR Paper Number 00-05. 
